\title{
Synthetic Environments as visualization method for product design
}

\author{
Frank Meijer*a ${ }^{\mathrm{a}}$ Egon L. van den Broek ${ }^{\tau a b c}$, Theo E. Schouten ${ }^{\mathrm{d}}$, Roy G.J. Damgrave ${ }^{\mathrm{a}}$, and \\ Huib de Ridder ${ }^{\mathrm{e}}$ \\ ${ }^{a}$ Departments of CPE/HMI/OPM, University of Twente, \\ P.O. Box 217, 7500 AE Enschede, The Netherlands \\ ${ }^{\mathrm{b}}$ Human-Centered Computing Consultancy (H-CCC), Vienna, Austria, \\ http://www.human-centeredcomputing.com/ \\ ${ }^{c}$ Karakter University Center, Radboud University Medical Center Nijmegen, \\ P.O. Box 9101, 6500 HB Nijmegen, The Netherlands \\ ${ }^{\mathrm{d}}$ Institute for Information Science (ICIS), Radboud University Nijmegen, \\ P.O. Box 9104, 6500 HE Nijmegen, The Netherlands \\ ${ }^{\mathrm{e}}$ Faculty of Industrial Design Engineering (IDE), Delft University of Technology, \\ Landbergstraat 15, 2628 CE Delft, The Netherlands
}

\begin{abstract}
In this paper, we explored the use of low fidelity Synthetic Environments (SE; i.e., a combination of simulation techniques) for product design. We explored the usefulness of low fidelity SE to make design problems explicit. In particular, we were interested in the influence of interactivity on user experience. For this purpose, an industrial design case was taken: the innovation of an airplane galley. A virtual airplane was created in which an interactive model of the galley was placed. First, three groups of participants explored the SE in different conditions: Participants explored the SE interactively (Interactive condition), watched a recording (Passive Dynamic condition), or watched static images (Passive Static condition). Afterwards, participants were tested in a questionnaire on how accurately they had memorized the spatial layout of the SE. The results revealed that interactive SE does not necessarily provoke participants to memorize spatial layouts more accurately. However, the effect of interactive learning is dependent on the participants' Visual Spatial Ability (VSA). Consequently, this finding supports use of interactive exploration of prototypes through low fidelity SE for the product design cycle when taking the individual's characteristics into account.
\end{abstract}

Keywords: interactive learning, virtual environments, product design, visual spatial abilities (VSA)

\section{INTRODUCTION}

Engineers and product designers learned by (bitter) experience that differences in backgrounds, interests, and paradigms can cause stakeholders to have different ideas (i.e., mental models ${ }^{1,2}$ ) of the product. This can cause huge problems in, for example, communication and cooperation and, consequently, might result in a disappointing product design. For the creative process of product design, designers traditionally use a drawing board and CAD software. However, recent technological developments enabled designers to employ new tools to support their creative process. In this paper, we explore the use of low fidelity Synthetic Environments (SE) to make specific design problems explicit.

The term "Synthetic Environments" refers to the use of a combination of simulation techniques, such as Virtual Reality (VR), Augmented Reality (AR), and Gaming technology to facilitate an artificial experience of certain situations ${ }^{3,4}$. These technologies intend to maximize the user's experience by realistically simulating a complex visual environment, possibly including sound, touch, or smell. The use of VR was originally mostly confined to military applications, for example to train soldiers for combat situations, because of the high costs involved with this type of technology.

*f.meijer@utwente.nl, frankmeij@gmail.com / ${ }^{\tau}$ vandenbroek@acm.org

Human Vision and Electronic Imaging XV, edited by Bernice E. Rogowitz, Thrasyvoulos N. Pappas, Proc. of SPIE-IS\&T Electronic Imaging, SPIE Vol. 7527, 752712 - (C) 2010 SPIE-IS\&T · CCC code:

0277-786X/10/\$18 - doi: $10.1117 / 12.843691$ 
However, the rapid development of computers in recent decennia has made it feasible for other application fields to use this type of technology as well. Henceforth, SE has become within reach for academic, commercial, industrial and educational domains ${ }^{5}$. We will focus on one of these domains specifically: industrial product design.

Due to the increased technological possibilities and decreased costs the use of SE has gained much interest of industrial companies to innovate their product design process. An SE provides several possibilities that traditional methods in product design do not facilitate. First, it visualizes a virtual context for the future use of a certain product. A (virtual) prototype can be tested in the earliest stages in its development on its effectiveness and usability ${ }^{6,7}$. Therefore, possible problems with the product are uncovered when adaptations are still easily made. Second, SE facilitates the involvement of end-users in the beginning of the design process, their opinions serving as an additional source of information for the designers ${ }^{8,9}$. Third, SE stimulates the communication between the different stakeholders in the design process ${ }^{10}$. Various stakeholders, such as designers, managers, or clients, who often use a different terminology, understand each other better with SE, not longer depending on the interpretation of separate images but on their experience with the (virtual) prototype.

For an effective application of low fidelity SE in the design process, there are two important constraints:

1) Financial: As for each new technique, $\mathrm{SE}$ have to show that they aid real-world design processes and, hence, save both time and money. To enable this, a SE should provide as much as possible the context that is relevant for the stakeholders; i.e., the amount of (visual) realism. Visual realism has shown to be an important factor for user experience ${ }^{11}$. Furthermore, visual realism increases visual spatial knowledge about the navigational space $^{12}$.

2) Interactivity: This characteristic is known to improve both user experience and spatial knowledge ${ }^{13,14}$. The current paper will investigate to what extent this characteristic is important for SE.

There is a large body of research that investigated why interactivity improves spatial knowledge of virtual environments. Christou and Bülthoff ${ }^{15}$ for example, showed that recognition of scenes in virtual environments is improved after interactive exploration as opposed to passive observation of an identical exploration. These researchers proposed that the user's possibility to control their viewpoints in the virtual environment contributes to more accurate mental representations in human visual spatial memory. James, Humphrey and Goodale ${ }^{16}$ reported a similar effect of interactivity for the exploration of unfamiliar 3D objects. These researchers showed that people who explored 3D objects interactively chose specific viewpoints that seemed most useful to them. They concluded that these viewpoints are essential to build up more complete mental representations. However, we found in prior experiments ${ }^{17,18}$ that the effect of interactivity on visual spatial memory is dependent on the user's Visual Spatial Ability (VSA). Users with a low VSA benefit from interactivity, whereas those with a high VSA do not. This finding indicates that users with a low VSA are not able to build up mental representations of 3D objects by passive observation, but are able to do so by interactive exploration. Users with a high VSA are able to build up object representations in both conditions. In summary, there is much evidence that interactivity enhances spatial knowledge, especially for users with a low VSA, because it enables them free control of their viewing direction in the virtual environment. We suggest that an improved spatial knowledge of virtual objects (i.e., prototypes) and environments (i.e., contexts) is the first important step to increase communication between the stakeholders and to reach consensus between them.

In the present study, we investigated the effect of interactivity and the influence of VSA on user experience and spatial knowledge of a low fidelity SE. We were interested whether the findings of previous research could be applied to an actual design case. For this purpose an SE was developed in which the specifications of the design case were used as input. The study was divided into three parts. 1) In a pre-test, the user's VSA was assessed. We expected that an effect of interactivity would be dependent of VSA. 2) In a learning phase, participants learned the spatial layout of the SE. In this part of the study, the possibility to interactively explore the SE was varied. Either the participants interactively explored the SE, observed a recording of another participant's interactive exploration, or viewed static images of the SE. The last condition was used as baseline condition. We expected that participants were more able to learn the spatial layout of the SE by interactive exploration, and were least able to learn it in the baseline condition. 3) In a test phase, participants received a questionnaire. The questionnaire assessed the participants' subjective experience of the spatial layout and the accuracy of the participants' mental representation of the SE. We expected that participants would show the highest scores in the interactive learning condition and the lowest scores in the baseline condition. 

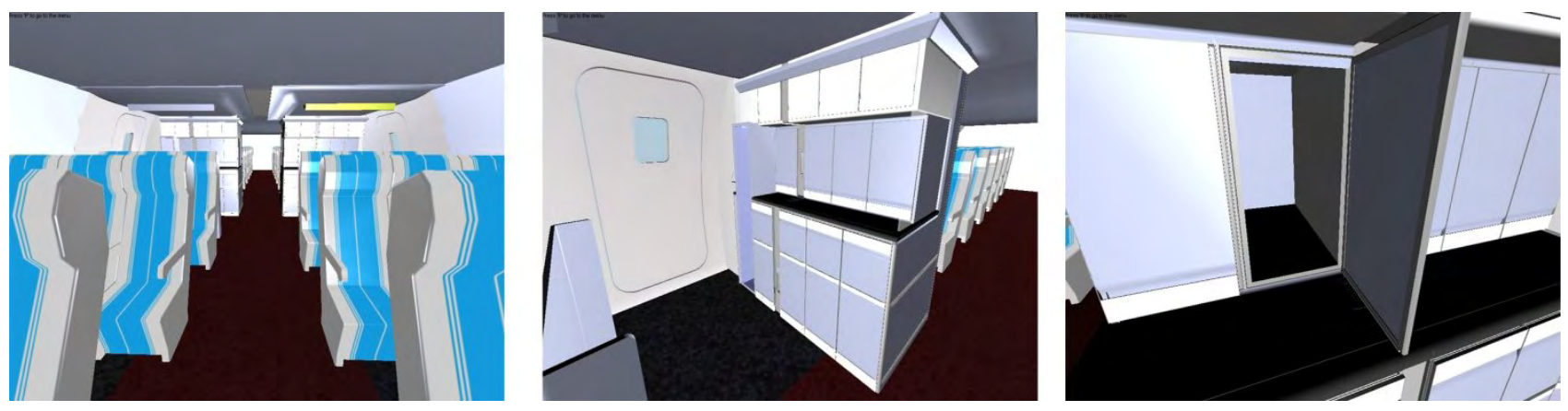

Figure 1. Images taken from the SE of the airplane cabin with the galley.

\section{METHODS}

\subsection{Participants}

Twelve participants (5 men and 7 women), aged 20-32 years (mean $=28.1$ ), were tested in the experiment. All had normal or corrected to normal vision and were naïve concerning the purposes of the experiment.

\subsection{The Synthetic Environment}

An industrial design case was used for the experiment: the innovation of an airplane galley. The design company Indes B.V. ${ }^{19}$ provided the case. An SE of an airplane cabin with the galley was developed with the 3D modeling software 3D Studio Max (Autodesk, Inc.) and Quest3D (Act-3D, B.V.). The SE was presented using a PC laptop (CPU: Intel Core2Duo 2.1GHz, Memory: 2GB) running Windows Vista (Microsoft, Inc.) connected to a 20-inch flat-screen monitor. A standard computer mouse and keyboard was used to walk through the SE. The arrow-keypad was used to walk from left to right and front to back. Holding the right mouse-button while moving the mouse enabled the participants to look around in the SE and to change the direction of the route. The left mouse-button was used to interact with the kitchen galley in the SE; a left button-click opened and closed the doors or moved the elevator up and down; see Figure 1 for an impression of the SE.

\subsection{Pre-test}

Before the experiment started, participants were assessed on their VSA; see also Figure 2. This was important, because in the test-phase of the experiment the participants were asked to recollect the spatial layout of the SE. Participants with a high VSA are more accurate on this task than those with a low VSA. Therefore, VSA was taken into account as a covariable in our data analysis.

To test the participants' VSA, the Vandenberg and Kuse's Mental Rotations Test (MRT-A) was used ${ }^{20,21}$. This test was used to determine to what degree participants were able to mentally rotate 3D objects. Participants compared an original object to four rotated alternatives and identified the two identical objects from them. In six minutes, participants completed as many of these comparisons as possible from a total of 24 comparisons. The percentage of correct comparisons determined the participant's test score and their VSA.

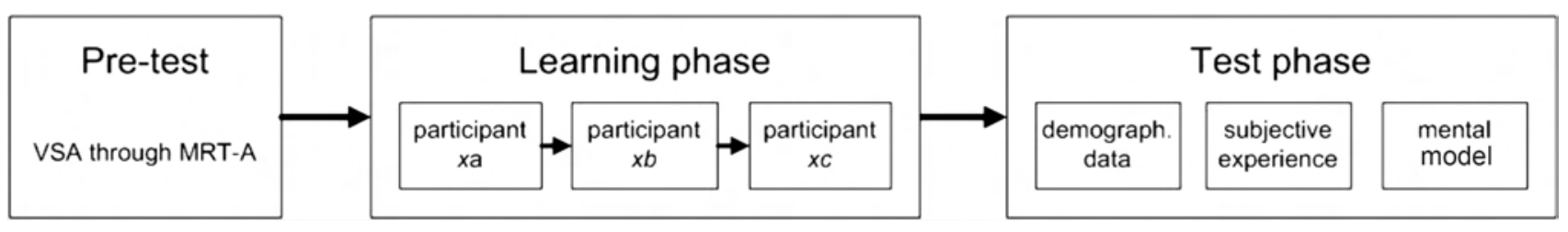

Figure 2. The three phases of the experiment, including its components. Moreover, the relation between the participants is depicted. 


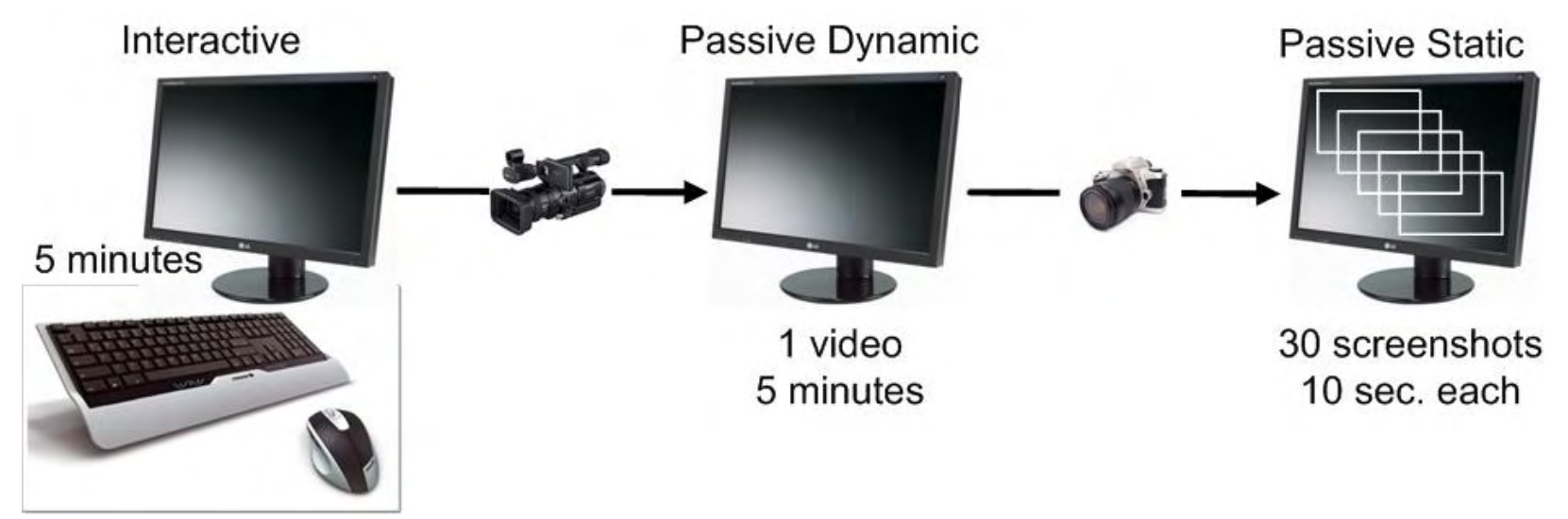

Figure 3. The three conditions of the learning phase, to which the participants were randomly assigned. Please note the relation between these conditions.

\subsection{Learning phase}

The learning phase consisted of three different learning conditions: an Interactive, a Passive Dynamic, and Passive Static learning condition; see also Figure 2 and 3. The participants were randomly assigned to one of these three conditions (i.e., four participants per condition). Prior to each of the conditions, participants were instructed to memorize the SE as accurately as possible, because they would subsequently be tested.

In the Interactive learning condition, the participants explored the SE freely with the mouse and keyboard; see also Figure 3. Participants were explicitly instructed to explore the kitchen galley by clicking on the galley doors and elevator. After 5 minutes the participants were instructed to stop. In this condition, the participants' actions were recorded and snapshots were taken each 10 seconds with the recording program FRAPS (Beepa, Inc.). This recording and these snapshots were used for the subsequent conditions. This was done to ensure that participants in the other two conditions observed identical viewpoints of the SE, and that only the type of learning was varied across participants.

In the Passive Dynamic learning condition, participants observed a recording taken from the prior participant in the Interactive condition, as is also shown in Figure 3. The procedure was identical to the Interactive condition, with the exception that participants were not able to interact with the SE in anyway. VLC media player software (VideoLAN, Org.) was used to present the recording.

In the Passive Static learning condition, participants viewed 30 snapshots taken from the video recorded of the participant in the prior Interactive condition, as is also shown in Figure 3. Each snapshot was presented for 10 seconds, which resulted in a total presentation time of five minutes. Again, participants were not able to interact with the SE in this condition. MS PowerPoint 2008 (Microsoft, Inc.) was used to present the snapshots.

\subsection{Test phase}

After the learning phase, participants were assessed in a questionnaire whether or not they had accurately memorized the spatial layout of the SE; see also Figure 2. The questionnaire was divided into three parts; for an overview of the questionnaire see Table 1 .

In the first part of the questionnaire demographic data was collected, such as the participants' age, gender, education, but also their affinity with computer games and 3D modeling. The latter two questions were used to control for the possibility that participants who are experienced with computer games or 3D modeling software more easily memorized the SE than those who are not ${ }^{22}$. Participants had to indicate whether they considered themselves as novice, advanced, or expert user. Furthermore, they indicated what type of games or 3D modeling software they used and how often. 
Table 1. Overview of the research variables with example questions used in the questionnaire.

\begin{tabular}{llll}
\hline Part & Research Variable & Example Question & $\begin{array}{c}\text { Type of } \\
\text { Measurement }\end{array}$ \\
\hline $\mathbf{1}$ & Demographics & What is your age, gender, education? & Open questions \\
& Gaming Experience & Do or did you play computer games? & Multiple choice \\
& 3D Modeling Experience & Do or did you 3D graphic software? & Multiple choice \\
\hline $\mathbf{2}$ & Spatial Memory & I can remember the spatial layout of the SE. & Likert Scale \\
& Survey Knowledge & I can remember the SE as a map. & Likert Scale \\
& Route Knowledge & I can remember the route that was walked through the SE. & Likert Scale \\
& Egocentric Representations & I had the feeling to move through the SE myself. & Likert Scale \\
& Ability to Imagine Actions & I know how to operate the airplane galley. & Likert Scale \\
\hline $\mathbf{3}$ & Size of the Cabin & What was the length, height, width of the cabin (in meters)? & Open question \\
& Size of the Galley & What was the length, height, width of the galley? & Open question \\
& Layout of the Galley & How many doors did the right, left galley have? & Open question \\
Orientation & Did the doors face to the back or to the front of the airplane? & Open question \\
\hline
\end{tabular}

The second part of the questionnaire assessed the participants' subjective experience of the SE. Five testing variables were distinguished: 1) To what extent participants had memorized the spatial layout of the SE in general, 2) the layout of the SE as a map, 3) the route through the SE, 4) to what extent they had identified themselves with the person walking through the SE, and 5) whether they were able to imagine to perform real actions with the airplane galley. Each variable comprised two questions. Thus, there were 10 questions in total this part. Answers were measured on a Likert scale from 1 to 5 , indicating the range from "I do not agree at all" to "I fully agree".

The third part of the questionnaire tested the participants' ability to reproduce the spatial layout of the SE. First, participants were required to provide size estimations of the airplane cabin in general, such as the cabin length, cabin width, cabin height, and path width. Second, participants estimated the size (height and width) of the two galleys. Third, they estimated the number of doors in the left and right galley. Fourth, they indicated the orientation of the galley and whether the doors faced the front or back of the airplane. This part of the questionnaire consisted of 10 questions in total.

\section{RESULTS}

Before the analyses were conducted, VSA, subjective experience, and estimation error scores were averaged per participant and per condition. Subsequently, the relative estimation errors $(\varepsilon)$ in $\%$ were calculated by dividing the absolute estimation error $(\delta)$ through the absolute estimated size $(\hat{S})$ in meters. Formally, this can be denoted as follows:

$$
\varepsilon=\frac{\delta}{\hat{S}} \cdot 100
$$


where $\delta=|\hat{s}-s|$ with $\mathrm{S}$ being the absolute size in meters (i.e., the size as envisioned for the SE) and $|\cdot|$ denotes the absolute value of . . Please note that the participants' estimation was taken as our ground truth and not the absolute size, as this is more or less arbitrary. This is easily shown when the equation above is rewritten as $\varepsilon=|1-s / \hat{s}| \cdot 100$. However, as this starting point can be judged as being rather unconventional, we have verified its possible influence on the results. Additional analyses revealed that similar results would have been obtained, when using the absolute size as ground truth.

After the relative estimation errors $\varepsilon$ were determined, various analyses were run to evaluate the data of the questionnaire. First, VSA was compared to the average scores of the subjective experience and the average relative estimation error $\varepsilon$. The results revealed that VSA in the Passive Dynamic condition was highest, followed by the Interactive condition, and was lowest in the Passive Static condition. Since we expected that VSA would have an impact on the effect of interactivity, we took these differences into account in our further analyses. The number of Gaming and/or 3D modeling experts was constant across conditions and did, therefore, not influence the effect of interactivity. As expected, participants showed the highest subjective experience scores in the Interactive condition, followed by the Passive Dynamic and Passive Static conditions. In contrast to our expectations, however, participants were least accurate estimating sizes in the Interactive condition; for a complete overview, see Table 2.

From the Subjective Experience - VSA plot it becomes evident that participants in the Interactive condition showed higher Likert scores than the other two conditions irrespective of VSA; see Figure 4 (left). Participants in the Passive Static condition all showed the lowest scores. Thus, the effect of interactivity seems to be robust for subjective experience even for a small group of participants. Furthermore, VSA did not seem to affect the subjective experience.

From the Estimation Error - VSA plot it becomes clear that the participants in the Passive Dynamic condition showed the lowest relative estimation errors $\varepsilon$, but also scored high on the VSA test; see Figure 4 (right). Participants in the Interactive condition showed the highest relative estimation errors $\varepsilon$. However, one clear exception to this is present in the interactive condition, with a relative estimation error $\varepsilon$ of $9.2 \%$ and a VSA score of 29 , as is shown in Figure 4 (right). Two participants of the Interactive condition showed respectively exceptional low and high relative estimation errors (i.e., respectively $\varepsilon=9.2 \%$ and $\varepsilon=37.6 \%$ ), when taking into account their VSA scores (i.e., respectively 29 and 50); see also Figure 4 (right). Thus, from this plot it becomes clear that the participants varied significantly in their relative estimation errors in the Interactive condition, an effect for which no simple (e.g., linear) relation was found with VSA.

To test the effect of interactivity on relative estimation error $\varepsilon$ and the influence of VSA, a linear best fit trend analysis was conducted with VSA (range: 0-100) and learning condition (Interactive, Passive Dynamic, Passive Static) as independent variables and the average relative estimation error $\varepsilon$ as dependent variable. The results of the linear best fit analysis of $[\varepsilon=a \cdot(100-\mathrm{VSA})+b \cdot$ condition $+c]$ showed that $a=0.16, b=-2.74$ en $c=16.8$, with a root mean square error $(r m s)$ of 8.5 . When the two participants were discarded who showed a large deviation on the scores, it showed $a=$ $0.30, b=-8.00$, and $c=21.3$ with a $r m s$ of 6.0 . This indicates that the relative estimation error $\varepsilon$ decreases with higher VSA scores and increases with interactivity.

Table 2. Overview of the average scores, as recorded for each of the learning conditions. Note that all values are percentages, except for the subjective experience scores.

\begin{tabular}{lllll}
\hline Learning Condition & VSA score & 3D/Gaming Experts & Subjective Experience & Estimation Error $(\varepsilon)$ \\
\hline 1. Interactive & 46 & 25 & 4.35 & 24.39 \\
2. Passive Dynamic & 66 & 25 & 4.00 & 13.52 \\
3. Passive Static & 34 & 25 & 3.35 & 20.69 \\
\hline
\end{tabular}



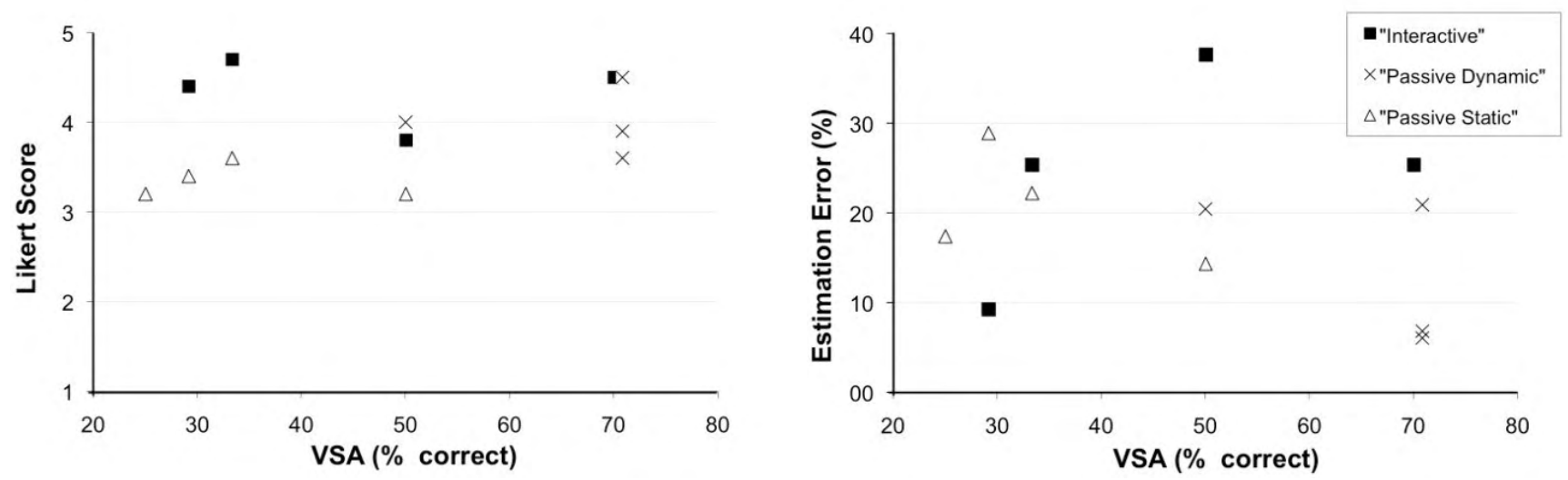

Figure 4. Plot of the average Likert scores (left) and relative estimation errors $\varepsilon$ (right) compared to VSA. Each condition is indicated separately and each data-point represents one participant.

Next, a more thorough comparison was made between the participants' subjective experience of the spatial layout of the $\mathrm{SE}$ in the three learning conditions. Overall, the results revealed the highest average scores in the Interactive condition, followed by the Passive Dynamic condition. The lowest scores were revealed in the Passive Static condition. These differences were most evident on three of the five research variables: the ability to remember the route walked through the SE, to form egocentric representations, and to imagine real actions with galley. Participants in the Passive Static condition scored lower those in the Passive Dynamic condition. Those in the Interactive condition scored highest. See Table 3 for an overview of the mean scores per research variable.

Finally, the participants' ability to reproduce the spatial layout of the SE by estimating the size of the airplane cabin, the airplane galley, the layout of the galley, and the orientation of the galley was analyzed. A repeated measures ANOVA on the accuracy data was run with estimation, 10× measured, as within subjects variable and learning condition (Interactive, Passive Dynamic, and Passive Static), gender (male, female), and VSA (scores: 25, 29(2x), 33(2x), 50(3x), 70, 71(3x)) as between subjects variables. The results revealed a main effect of Learning condition, Learning condition $F(2,1)=$ 334.0, $p<.04$. The participants were more accurate in the Passive Dynamic condition as compared to the Passive Static condition. Against our expectations, participants in the Interactive condition were least accurate estimating the spatial layout of the SE. Furthermore, a main effect of VSA score was found, $F(4,1)=172.1, p=.06$ (one-tailed). Participants with high VSA were more accurate estimating than those with a low VSA. The interaction between learning condition and VSA was also significant, $F(3,1)=375.8, p=.04$. Also, a significant main effect was found of gender, $F(1,1)=$ $553.5, p=.03$. Male participants performed better than the female participants, which is in line with other research ${ }^{23}$. See Table 4 for an overview of the mean accuracy of estimating the spatial layout of the SE.

Table 3. Overview of the participants' subjective experience of the spatial layout of the SE; i.e., part 2 of the questionnaire.

\begin{tabular}{llllll}
\hline Learning Condition & $\begin{array}{l}\text { Spatial } \\
\text { Memory }\end{array}$ & $\begin{array}{l}\text { Survey } \\
\text { Knowledge }\end{array}$ & $\begin{array}{l}\text { Route } \\
\text { Knowledge }\end{array}$ & $\begin{array}{l}\text { Egocentric } \\
\text { Representations }\end{array}$ & $\begin{array}{l}\text { Imagining } \\
\text { Actions }\end{array}$ \\
\hline 1. Interactive & 4.63 & 4.63 & 4.50 & 3.63 & 4.38 \\
2. Passive Dynamic & 4.75 & 4.38 & 4.00 & 3.13 & 3.75 \\
3. Passive Static & 3.75 & 4.13 & 3.75 & 2.50 & 2.63 \\
\hline
\end{tabular}


Table 4. Overview of the participants' error in estimating the spatial layout of the SE; i.e., part 3 of the questionnaire.

Table cells represent the mean error from the values used in the SE in percentages.

\begin{tabular}{lllll}
\hline Learning Condition & Cabin Size & Galley Size & Galley Layout & Orientation \\
\hline 1. Interactive & 29.0 & 28.7 & 16.2 & 0.00 \\
2. Passive Dynamic & 19.9 & 17.7 & 4.30 & 0.00 \\
3. Passive Static & 18.0 & 21.1 & 31.0 & 0.00 \\
\hline
\end{tabular}

\section{DISCUSSION}

In an experiment, we investigated the effect of interactivity on user experience and spatial knowledge of a low fidelity SE. An SE was developed of an actual design case: the innovation of an airplane galley. Participants learned the SE either in an Interactive, Passive Dynamic, or Passive Static learning condition. In the Interactive condition, they were able to explore the SE freely with a computer mouse and keyboard. In the Passive Dynamic condition, the participants observed a recording and in the Passive Static snapshots of another participants' exploration of the SE. Afterwards they were tested on their subjective experience and the accuracy of their mental representation of the spatial layout of the airplane cabin and galley. We also investigated the influence of VSA. We expected that participants would improve their subjective experience and the accuracy of their mental representations in the Interactive condition compared to the Passive Dynamic and the Passive Static condition. We expected poorest scores and accuracy in the Passive Static condition. Furthermore, we expected that the effect of interactivity would be dependent of participants' VSA.

The results partly confirmed these expectations. As expected, participants in the Interactive condition showed the highest average scores on the subjective experience compared to the other two conditions. Those in the Passive Static showed the lowest. Differences between the conditions were largest on three of the five research variables of this part of the questionnaire: acquisition of route knowledge, acquisition of egocentric representations, and imagining actions. In the Interactive condition, participants indicated more strongly that they remembered the route through the airplane cabin, sensed to have explored the SE themselves, and were able to imagine real actions with the airplane galley.

In contrast to our expectations, participants in the Interactive condition were not more accurate when they had to reproduce the spatial layout of the SE. When participants had to estimate sizes of the airplane cabin and galley, they were in fact least accurate in the Interactive and most accurate in the Passive Dynamic condition. One possible explanation for this unexpected effect is the average VSA score in the conditions: Average VSA scores were highest in the Passive Dynamic conditions. However, a linear best fit analysis of Learning condition and VSA showed that not only estimation accuracy improved with higher VSA scores, but also with less dynamic conditions (respectively: Interactive, Passive Dynamic, and Passive Static). Thus, the impaired performance of participants in the Interactive condition cannot merely be explained by VSA. This is in contrast with prior research ${ }^{14-18}$.

Another possible explanation for this result is that the camera position in the Interactive and Passive conditions is continually changed, which can make it difficult for participants to get a precise idea about the size of the SE. In the Passive Static condition the participants observed still images of the SE each for 10 seconds. This period can be used to fully focus on the size of the SE. Christou and Bülthof ${ }^{15}$ provided evidence that the viewpoints taken in a virtual environment determine the accuracy of the mental representations built from them. Future research should take into account that the time these viewpoints last also is of influence. Furthermore, the difference between the Interactive and Passive Dynamic conditions is possibly explained by the fact that participants in the Interactive condition concentrate more on performing actions than on the spatial layout of the SE. In fact, the participants were explicitly instructed to interactively explore the airplane galley. In the Passive Dynamic condition participants did not receive this instruction so they were able to fully focus on the spatial layout of the SE. This possibility is supported by the finding that participants in the Interactive condition indicated to be more able to imagine actions with the product than in the Passive Dynamic condition.

In sum, we did not find that interactivity improved visual spatial memory directly. In contrast to prior research we found the opposite effect: Participants performed poorly estimating the spatial layout of the SE. We suggested that there are a number of unknown factors that may have modulated the effect of interactivity. Future research should further investigate these factors. The present study did confirm, however, that interactivity does improve the participants' 
subjective experience of SE. In particular, the ability to imagine actions with the virtual prototype in SE was improved after interactive exploration. Therefore, we conclude that interactive exploration of prototypes through SE improves the stakeholders' mental models of product design especially about its practical functionality.

\section{ACKNOWLEDGMENTS}

The authors gratefully acknowledge the support of the Dutch Innovation Oriented Research Program 'Integrated Product Creation and Realization (IOP-IPCR)' of the Dutch Ministry of Economic Affairs. Furthermore, we thank Mascha C. van der Voort (Laboratory of Design, Production, and Management, University of Twente) and Joris S.M. Vergeest (Industrial Design Engineering, Delft University of Technology) for their efforts as project coordinators. Last, we specifically thank Willem Mees van der Bijl and Niek Wellink from Indes B.V. for making this research possible.

\section{REFERENCES}

1. Pylyshyn, Z. W., "What the mind's eye tells the mind's brain," Psychological Bulletin 80(1), 1-24 (1973).

2. Johnson-Laird, P. N., "Mental models in cognitive science," Cognitive Science 4(1), 71-115 (1980).

3. Wang, H., Vergeest, J. S. M., Miedema, J., Meijer, F., Van der Voort, M. C. Van den Broek, E. L., "Synthetic Environment as a communication tool for Dynamic Prototyping," Proc. of the 6th International Seminar and Workshop Engineering Design in Integrated Product Development (EDIProD), 211-219 (2008).

4. Wang, H., Meijer, F., Miedema, J., Broek, E.L. van den, Voort, M.C. van der, and Vergeest, J.S.M., "Applicability of Synthetic Environments for Product Design". In X. Fischer and P. Ray (Eds.), Integrated Design and Manufacturing in Mechanical Engineering (series: Research in Interactive Design, Vol. 3). Springer-Verlag. [in press]

5. Burdea, G. C., Coiffet. P., "Virtual Reality Technology”, MIT, New York (2003).

6. Van den Broek, E. L., Meijer, F., Miedema, J., Wang, H., Van der Voort, M. C., and Vergeest, J. S. M., "Synthetic Environments for cooperative product design," Lecture Notes in Computer Science (Cooperative Design, Visualization and Engineering) 5220, 1-10 (2008).

7. Smulders, F.E., Broek, E.L. van den, and Voort, M.C. van der, "A socio-interactive framework for the fuzzy front end," In A. Fernandes, A. Teixeira, and R. Natal Jorge (Eds.), Proc. of the 14th International Product Development Management Conference, 1439-1450 (2007).

8. Tideman, M., Van der Voort, M. C., and Van Houten, F. J. A. M., "A new product design method based on Virtual Reality, Gaming and Scenarios," Int. Journal on Interactive Design and Manufacturing 2(4), 195-205 (2008).

9. Tideman, M., Van der Voort, M. C., Van Houten, F. J. A. M., (2005). Using Virtual Reality for Scenario Based Product Design. In Proceedings of Virtual Concept, Biarritz - France, 2005.

10. Landsman, R.B., Broek, E.L. van den, \& Gieskes, J.F.B. (2009). Creating shared mental models: The support of visual language. Lecture Notes in Computer Science (Cooperative Design, Visualization and Engineering), 5738, 161-168.

11. Slater, M., K"hanna, P., Mortensen, J., Yu, I., "Visual realism enhances realistic response in an immersive virtual environment, IEEE Computer Graphics and Applications 29(3), 76-84 (2009).

12. Meijer, F., Geudeke, B. L., and Van den Broek, E. L., "Navigating through virtual environments: Visual realism improves spatial cognition," CyberPsychology and Behavior 12(5), 517-521 (2009).

13. Larsson, P., Västfjäll, D., and Kleiner, M., "The actor-observer effect in virtual reality presentations," CyberPsychology and Behavior 4(2), 239-246 (2007).

14. Farell, M. J., Arnold, P., Pettifer, S., Adams, J., Graham, T., and MacManamon, M., "Transfer of route learning from virtual to real environments," Journal of Experimental Psychology: Applied 9(4), 219-227 (2003).

15. Christou, C. G., and Bülthoff, H. H., "View dependence in scene recognition after active learning," Memory and Cognition 27(6), 996-1007 (1999). 
16. James, K. H., Humphrey, K., and Goodale, M. A., "Manipulating and recognizing virtual objects: where the action is," Canadian Journal of Experimental Psychology 55(2), 111-120 (2001).

17. Meijer, F., Broek, E.L. van den., and Schouten, Th.E., "The impact of interactive manipulation on the recognition of objects," Proc. of SPIE (Human Vision and Electronic Imaging XIII), 6806, 68060H-68060H-7 (2008).

18. Meijer, F. and Van den Broek, E. L., "Representing 3D virtual objects: Evidence for an interaction between visuospatial ability and the nature of exploration," Vision Research, 50(x), xxx-xxx [in press].

19. Indes B.V.; URL: http://www.indes.eu/. [Last accessed: January 13, 2010]

20. Vandenberg, S. G., and Kuse, A. R., "Mental rotations, a group test of three-dimensional spatial visualization," Perceptual and Motor Skills 47(2), 599-601 (1978).

21. Peters, M., Laeng, B., Latham, K., Jackson, M., Zaiyouna, R., and Richardson, C., "A redrawn Vandenberg and Kuse mental rotations test: Different versions and factors that affect performance," Brain and Cognition 28(1), 3958 (1995).

22. Epstein, R.A., Higgins, J.S., and Thompson-Schill, S.L., "Learning places from views: Variation in scene processing as a function of experience and navigational ability," Journal of Cognitive Neuroscience 17(1), 73-83 (2005).

23. Hirnstein M., Bayer, U., and Hausmann, M., "Sex-specific response strategies in mental rotation," Learning and Individual Differences 19(2), 225-228 (2009). 